in 87 children born to mothers positive for anti-Ro/SSA autoantibodies, from a single Canadian center.

All children underwent a full clinical examination (which included head circumference measurement) at each clinic visit. If the head circumference was enlarged compared to that of age-standardized normal values, cerebral imaging (ultrasonography or CT) was performed.

Of the 87 infants, 47 were diagnosed with NLE. One infant with NLE required neurosurgical intervention. The majority of abnormally large head circumference measurements were observed in children aged between 8 and 24 months. Owing to a rapid increase in head circumference, or a head circumference percentile disproportionately larger than height and weight percentiles, 13 children underwent cerebral imaging. Of these 13 children, 7 had hydrocephalus, 5 of whom also had NLE. The overall prevalence of hydrocephalus in this cohort was $8 \%$ (which is higher than that observed in the general population) with all infants diagnosed between 2 and 11 months of age.

The authors suggest that hydrocephalus is a late-appearing clinical manifestation of NLE and children of anti-Ro/SSA autoantibodypositive mothers should be monitored for macrocephaly and hydrocephalus.

Original article Boros CA et al. (2007) Hydrocephalus and macrocephaly: new manifestations of neonatal lupus erythematosus. Arthritis Rheum 57: 261-266

\section{Bisphosphonates are cost- effective in steroid-treated patients with high fracture risk}

Glucocorticoid-induced fracture can be prevented with bisphosphonate therapy, but data on the cost-effectiveness of this practice are scarce. Van Staa and colleagues, therefore, evaluated the cost-effectiveness of bisphosphonates in reducing fractures in patients who receive glucocorticoid therapy.

Their cost-efficacy model was based on data from 191,752 UK patients aged $\geq 40$ years who were prescribed daily oral glucocorticoids, 9,259 of whom experienced fracture. Age, sex, glucocorticoid daily and cumulative dose, and indication were used to calculate fracture and mortality risks. Costs of bisphosphonate treatment were estimated per quality-adjusted life-year gained as a result of fracture prevention, and per number of fractures prevented, in patients who received glucocorticoids for 5 years.

Bisphosphonate costs per fracture avoided in women were UK£17,000 with daily $5 \mathrm{mg}$ glucocorticoid use (£27,000 in men) and $£ 10,000$ with daily $15 \mathrm{mg}$ glucocorticoid use $(£ 18,000$ in men). The costs per quality-adjusted lifeyear gained varied with life expectancy, but the cost per fracture did not. Glucocorticoid dose and indication, patient age, and sex all strongly influenced the cost of fracture prevention. Bisphosphonates were most cost-effective in patients with rheumatoid arthritis, and were more cost-effective in patients with a low $\mathrm{BMI}\left(<20 \mathrm{~kg} / \mathrm{m}^{2}\right)$ than in patients with a high BMI $\left(\geq 26 \mathrm{~kg} / \mathrm{m}^{2}\right)$.

The authors conclude that bisphosphonate therapy is cost-effective in glucocorticoidtreated patients at a high risk of fracture - elderly patients with a life expectancy of $>5$ years, individuals with a history of fracture, rheumatoid arthritis, low BMI, or those receiving high glucocorticoid doses.

Original article van Staa TP et al. (2007) Individual fracture risk and the cost-effectiveness of bisphosphonates in patients using oral glucocorticoids. Rheumatology (Oxford) 46: 460-466

\section{Positive results for rituximab in patients with neuropsychiatric SLE}

Involvement of the central nervous system (CNS) in patients with systemic lupus erythematosus (SLE) is associated with poor prognosis. In many patients, neuropsychiatric SLE is resistant to treatment, but no established management protocol for this condition exists. Tokunaga and colleagues have now demonstrated that rituximab might be an effective treatment for refractory neuropsychiatric SLE.

This noncontrolled Japanese study included 10 female patients with neuropsychiatric SLE. The patients had various CNS symptoms, including acute confusion, psychosis, seizures and mood disorders, which were resistant to conventional treatment. All patients received moderate-to-high doses of corticosteroids, and different dosing regimens of rituximab (e.g. $375 \mathrm{mg} / \mathrm{m}^{2}$ once a week for 2 weeks). Immunosuppressive treatment was discontinued in all except one patient who continued to receive azathioprine. The follow-up period was 7-45 months. 